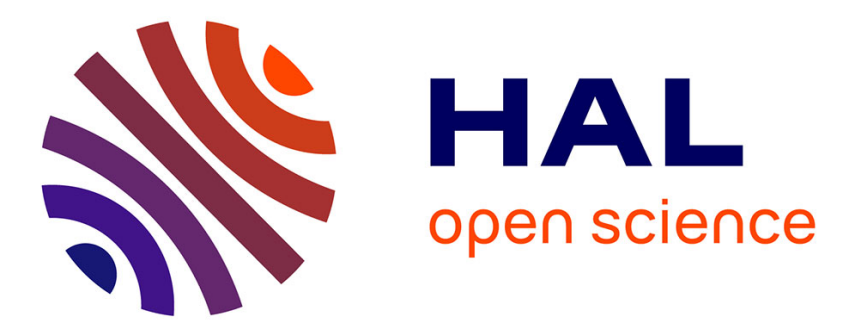

\title{
Dynamic Identification of a 6 Dof Robot without Joint Position Data
}

\author{
M. Gautier, Pierre Olivier Vandanjon, A. Janot
}

\section{To cite this version:}

M. Gautier, Pierre Olivier Vandanjon, A. Janot. Dynamic Identification of a 6 Dof Robot without Joint Position Data. 2011 IEEE International Conference on Robotics and Automation (ICRA 2011), May 2011, France. pp 234-239. hal-00638032

\section{HAL Id: hal-00638032 https://hal.science/hal-00638032}

Submitted on 3 Nov 2011

HAL is a multi-disciplinary open access archive for the deposit and dissemination of scientific research documents, whether they are published or not. The documents may come from teaching and research institutions in France or abroad, or from public or private research centers.
L'archive ouverte pluridisciplinaire HAL, est destinée au dépôt et à la diffusion de documents scientifiques de niveau recherche, publiés ou non, émanant des établissements d'enseignement et de recherche français ou étrangers, des laboratoires publics ou privés. 


\title{
Dynamic identification of a 6 dof robot without joint position data
}

\author{
M. Gautier ${ }^{(1)}$, P-O Vandanjon ${ }^{(2)}$ and A. Janot ${ }^{(3)}$
}

(1) Université de Nantes, IRCCyN, 1, rue de la Noë - BP 92101 - 44321 Nantes Cedex 03, France

(2) Laboratoire Central des Ponts et Chaussées, Route de Bouaye BP 4129, 44341Bouguenais, France

(3) ONERA DCSD, 2 Avenue Edouard Belin 31055 - BP 74025 - Toulouse Cedex 04, France

\begin{abstract}
Off-line robot dynamic identification methods are mostly based on the use of the inverse dynamic model, which is linear with respect to the dynamic parameters. This model is calculated with torque and position sampled data while the robot is tracking reference trajectories that excite the system dynamics. This allows using linear least-squares techniques to estimate the parameters. This method requires the joint force/torque and position measurements and the estimate of the joint velocity and acceleration, through the bandpass filtering of the joint position at high sampling rates. A new method called DIDIM (Direct and Inverse Dynamic Identification Models) has been proposed and validated on a 2 degree-offreedom robot [1]. DIDIM method requires only the joint force/torque measurement. It is based on a closed-loop simulation of the robot using the direct dynamic model, the same structure of the control law, and the same reference trajectory for both the actual and the simulated robot. The optimal parameters minimize the 2-norm of the error between the actual force/torque and the simulated force/torque. A validation experiment on a 6 dof Staubli TX40 robot shows that DIDIM method is very efficient on industrial robots.
\end{abstract}

\section{INTRODUCTION}

$\mathrm{T}$ HE usual identification method based on the inverse dynamic identification model and least-squares (IDIMLS) technique has been successfully applied to identify inertial and friction parameters of several robotic prototypes and industrial robots [2], [3], [4], [5], [6], [7], [8], [9] , amongst others. Good results can be obtained provided a well-tuned derivative bandpass filtering of joint position to calculate the joint velocities and accelerations is used.

The Direct and Inverse Dynamic Identification Models (DIDIM) method needs only the joint force/torque measurements [1]. It is based on a closed-loop simulation using the direct dynamic model while the optimal parameters minimize the 2-norm of the error between the actual force/torque and the simulated force/torque, assuming the same control law and the same reference trajectory. In spite it is difficult to know details on the control law for most industrial robots, it is possible to get this information from collaboration with manufacturers who want to improve the performances of their robots. This non-linear least-squares problem is dramatically simplified using the inverse dynamic model to formulate the simulated force/torque as an algebraic function linear in relation to the parameters. This paper recalls the DIDIM method and gives new experimental results obtained using a 6 dof robot.

The paper is organized as follows: section II reviews the usual identification technique of the dynamic parameters of the robot. Section III presents the DIDIM method. The modeling of the TX40 industrial robot is presented in sectionIV. The experimental results are given in section $\mathrm{V}$. Finally, section VI is the conclusion.

\section{IDIM: INVERSE DYNAMIC IDENTIFICATION MODEL}

\section{TECHNIQUE}

Identification results obtained with the IDIM method are compared with those obtained with the new DIDIM method. Moreover, the IDIM method is used at each step of the iterative procedure in DIDIM. So it is important to give a review of the conventional IDIM method.

The inverse dynamic model (IDM) of a rigid robot composed of $n$ moving links calculates the motor torque vector $\tau_{i d m}$, as a function of the generalized coordinates and their derivatives. It can be obtained from the Newton-Euler or the Lagrangian equations [10]. It is given by:

$\tau_{\text {idm }}=M(q) \ddot{q}+N(q, \dot{q})$

Where $q, \dot{q}$ and $\ddot{q}$ are respectively the $(n \times 1)$ vectors of generalized joint positions, velocities and accelerations, $M(q)$ is the $(n \times n)$ robot inertia matrix, and $N(q, \dot{q})$ is the $(n \times 1)$ vector of centrifugal, Coriolis, gravitational and friction forces/torques. The modified Denavit and Hartenberg notation allows obtaining a dynamic model that is linear in relation to a set of standard dynamic parameters, $\chi_{s t},[4]:$

$\tau_{i d m}=I D M_{s t}(q, \dot{q}, \ddot{q}) \chi_{s t}$

Where $I D M_{s t}(q, \dot{q}, \ddot{q})$ is the $\left(n \times N_{s}\right)$ jacobian matrix of $\tau_{i d m}$, with respect to the $\left(N_{s} \mathrm{x} 1\right)$ vector $\chi_{s t}$ of the standard 
parameters given by $\chi_{s t}=\left[\begin{array}{lllll}\chi_{s t}^{l \mathrm{~T}} & \chi_{s t}^{2 \mathrm{~T}} & \cdots & \chi_{s t}^{n \mathrm{~T}}\end{array}\right]^{\mathrm{T}}$ :

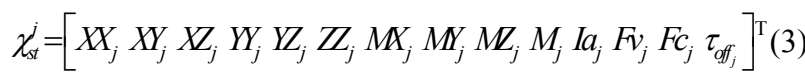

where: $X X_{j}, X Y_{j}, X Z_{j}, Y Y_{j}, Y Z_{j}, Z Z_{j}$ are the six components of the inertia matrix of link $j$ at the origin of frame $j . M X_{j}, M Y_{j}, M Z_{j}$ are the components of the first moment of link $j . M_{j}$ is the mass of link $j, I a_{j}$ is a total inertia moment for rotor and gears of actuator $j . F v_{j}$, $F c_{j}$ are the viscous and Coulomb friction parameters of joint $j . \tau_{o f f_{j}}$ is an offset parameter.

The base parameters are the minimum number of dynamic parameters from which the dynamic model can be calculated. They are obtained from the standard inertial parameters by regrouping some of them by means of linear relations [11]. The minimal inverse dynamic model can be written as:

$\tau_{i d m}=\operatorname{IDM}(q, \dot{q}, \ddot{q}) \chi$

$\operatorname{IDM}(q, \dot{q}, \ddot{q})$ is the $(n \times b)$ matrix of the minimal set of basis functions of the rigid body dynamics,

$\chi$ is the $(b \times 1)$ vector of the $b$ base parameters.

Because of perturbations due to noise measurement and modeling errors, the actual force/torque $\tau$ differs from $\tau_{i d m}$ by an error, $e$, such that:

$\tau=\tau_{i d m}+e=\operatorname{IDM}(q, \dot{q}, \ddot{q}) \chi+e$

Equation (6) represents the Inverse Dynamic Identification Model (IDIM). We consider the off-line identification of the base dynamic parameters $\chi$, given measured or estimated off-line data for $\tau$ and $(q, \dot{q}, \ddot{q})$, collected while the robot is tracking some planned trajectories.

$(q, \dot{q}, \ddot{q})$ in (6) are estimated with $(\hat{q}, \hat{\dot{q}}, \hat{\dot{q}})$, respectively, obtained by bandpass filtering the measure of $q$ [7]. The actual force/torque, $\tau$ is calculated by:

$\tau=g_{\tau} v_{\tau}$

where $v_{\tau}$ is the $(n \times 1)$ control signal vector calculated according to the control law and $g_{\tau}$, is the $(n \times n)$ diagonal matrix of the drive gains.

The inverse dynamic identification model (IDIM) (6) is sampled at a frequency measurement $f_{m}$, at different times $t_{k}, k=1, \ldots, n_{m}$, while the robot is tracking a reference trajectory $\left(q_{r}, \dot{q}_{r}, \ddot{q}_{r}\right)$, during the time length $T_{o b s}$, of the trajectory.

We obtain an over determined linear system of $n^{*} T_{o b s} * f_{m}$ equations and $b$ unknowns such that:

$Y_{f m}(\tau)=W_{f m}(\hat{q}, \hat{\dot{q}}, \hat{\dot{q}}) \chi+\rho_{f m}$
In order to window the identification frequency range into the model dynamics, a parallel decimation procedure lowpass filters in parallel $Y_{f m}$ and each column of $W_{f m}$ and resamples them at a lower rate, keeping one sample over $n_{d}$. We obtain:

$$
Y(\tau)=W(\hat{q}, \hat{\dot{q}}, \hat{\dot{q}}) \chi+\rho
$$

where: $Y(\tau)$ is the $(r \times 1)$ vector of measurements, built from the actual force/torque $\tau$. $W(\hat{q}, \hat{\dot{q}}, \hat{\ddot{q}})$ is the $(r \mathrm{x} b)$ observation matrix, built from the estimated values $(\hat{q}, \hat{\dot{q}}, \hat{\ddot{q}})$ of $(q, \dot{q}, \ddot{q}) . \rho$ is the $(r \times 1)$ vector of errors. $r=n * n_{m} / n_{d}$ is the number of rows in (9). In $Y$ and $W$, the equations of each joint are grouped together such that:

$Y=\left[\begin{array}{lll}\left(Y^{l}\right)^{T} & \ldots & \left(Y^{n}\right)^{T}\end{array}\right]^{T}, W=\left[\begin{array}{lll}\left(W^{l}\right)^{T} & \ldots & \left(W^{n}\right)^{T}\end{array}\right]^{T}$

$Y^{j}$ and $W^{j}$ represent the $n_{m} / n_{d}$ equations of joint $j$. The ordinary LS (OLS) solution $\hat{\chi}$ minimizes $\|\rho\|^{2}$. Using the base parameters and tracking "exciting" reference trajectories [12], we get a well conditioned matrix $W$. The LS solution $\hat{\chi}$ is given by:

$\hat{\chi}=\left(\left(W^{T} W\right)^{-1} W^{T}\right) Y=W^{+} Y$

Standard deviations $\sigma_{\hat{\chi}_{i}}$, are estimated under the assumptions that $W$ is a deterministic matrix and $\rho$, is a zero-mean additive independent Gaussian noise, with a covariance matrix $C_{\rho \rho}$, such that:

$C_{\rho \rho}=E\left(\rho \rho^{\mathrm{T}}\right)=\sigma_{\rho}^{2} I_{\mathrm{r}}$

$E$ is the expectation operator and $I_{r}$, the $r x r$ identity matrix. An unbiased estimation of the standard deviation $\sigma_{\rho}$ is:

$\hat{\sigma}_{\rho}^{2}=\|Y-W \hat{\chi}\|^{2} /(\mathrm{r}-\mathrm{b})$

The covariance matrix of the estimation error is given by:

$C_{\hat{\chi} \hat{\chi}}=E\left[(\chi-\hat{\chi})(\chi-\hat{\chi})^{\mathrm{T}}\right]=\hat{\sigma}_{\rho}^{2}\left(W^{\mathrm{T}} W\right)^{-1}$

$\sigma_{\hat{\chi}_{i}}^{2}=\mathrm{C}_{\hat{\chi} \hat{x}}(i, i)$ is the $i^{\text {th }}$ diagonal coefficient of $C_{\hat{\chi} \hat{x}}$. The relative standard deviation $\% \sigma_{\hat{\chi}_{\mathrm{ri}}}$ is given by:

$\% \sigma_{\hat{\chi}_{\mathrm{ri}}}=100 \sigma_{\hat{\chi}_{\mathrm{i}}} /\left|\hat{\chi}_{\mathrm{i}}\right|$, for $\left|\hat{\chi}_{\mathrm{i}}\right| \neq 0$

The OLS can be improved by taking into account different standard deviations on joint $j$ equations errors [7]. Each equation of joint $j$ in (9), (10), is weighted with the inverse of the standard deviation of the error calculated from OLS solution of the equations of joint $j$, given by:

$Y^{j}\left(\tau_{j}\right)=W^{j}\left(I D M^{j}(\hat{q}, \hat{\dot{q}}, \hat{\ddot{q}})\right) \chi+\rho^{j}$

This weighting operation normalises the errors in (9) and gives the weighted LS (WLS) estimation of the parameters.

This identification method is illustrated in Fig. 1. 


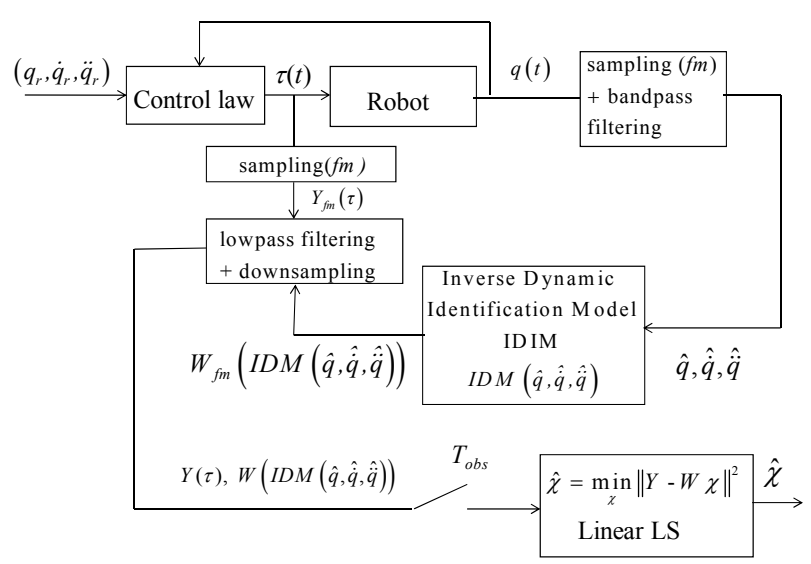

Fig. 1. IDIM LS identification scheme.

\section{DIDIM: DIRECT AND INVERSE DYNAMIC IDENTIFICATION MODEL TECHNIQUE}

\section{A. Theoretical approach}

DIDIM [1] is a closed loop output error (CLOE) method which does not require joint position data. The output, $y=\tau$, is the actual joint force/torque $\tau$, and the simulated output $y_{s}=\tau_{d d m}$, is the simulated joint force/torque. $\tau_{d d m}$, is the force/torque input of the Direct Dynamic Model (DDM) which can be obtained by writing the IDM equation (1), as following:

$M\left(q_{d d m}, \chi\right) \ddot{q}_{d d m}=\tau_{d d m}-N\left(q_{d d m}, \dot{q}_{d d m}, \chi\right)$

Where $M\left(q_{d d m}, \chi\right)$ and $N\left(q_{d d m}, \dot{q}_{d d m}, \chi\right)$ depend on an estimation of the base parameters $\chi$.

The signal $q_{d d m}(t, \chi)$, is the result of the integration of the linear implicit differential equation. The optimal solution, $\hat{\chi}$, minimizes the quadratic criterion, $C(\chi)=\left\|Y_{s}-Y\right\|^{2}$.

$Y(\tau)$ and $Y_{S}\left(\tau_{d d m}\right)$ are vectors obtained by filtering and downsampling the vectors of samples of the actual force/torque $\tau$, and of the simulated force/torque $\tau_{d d m}$, respectively.

This non-linear LS problem is solved by the GaussNewton regression. It is based on a Taylor series expansion of $y_{s}$, at a current estimate $\hat{\chi}^{k}$, of the parameters at iteration $k$ :

$y_{S}\left(\chi^{k+1}\right)=y_{S}\left(\hat{\chi}^{k}\right)+\left(\partial\left(y_{S}(\chi)\right) / \partial \chi\right)_{\hat{\chi}^{k}}\left(\chi^{k+1}-\hat{\chi}^{k}\right)+o$

$\left(\partial\left(y_{S}(\chi)\right) / \partial \chi\right)_{\hat{\chi}^{k}}$ is the $(n \times b)$, jacobian matrix of $y_{s}$, with respect to $\chi$, evaluated at $\hat{\chi}^{k}$. The input force/torque of the DDM, $\tau_{d d m}$, can be calculated with the analytical expression of the inverse dynamic model (4), such as:

$y_{s}(\chi)=\tau_{d d m}(\chi)=\tau_{\text {idm }}(\chi)=\operatorname{IDM}\left(q_{d d m}(\chi), \dot{q}_{d d m}(\chi), \ddot{q}_{d d m}(\chi)\right) \chi$

Then the jacobian matrix is given by:

$$
\begin{aligned}
\left(\frac{\partial\left(y_{S}\right)}{\partial \chi}\right)_{\hat{\chi}^{k}} & =\left(\frac{\partial\left(\tau_{d d m}\right)}{\partial \chi}\right)_{\hat{\chi}^{k}}=\left(\frac{\partial\left(\tau_{i d m}\right)}{\partial \chi}\right)_{\hat{\chi}^{k}} \\
& =\frac{\partial}{\partial \chi}\left(I D M\left(q_{d d m}\left(\hat{\chi}^{k}\right), \dot{q}_{d d m}\left(\hat{\chi}^{k}\right), \ddot{q}_{d d m}\left(\hat{\chi}^{k}\right)\right) \hat{\chi}^{k}\right)
\end{aligned}
$$

Because of the same closed loop control for the actual and for the simulated robot (see section B), the simulated position, velocity and acceleration have little dependence on $\chi$ and remains close to the actual ones for any $\hat{\chi}_{k}$ :

$\left(q_{d d m}\left(\hat{\chi}_{k}\right), \dot{q}_{d d m}\left(\hat{\chi}_{k}\right), \ddot{q}_{d d m}\left(\hat{\chi}_{k}\right)\right) \simeq(q, \dot{q}, \ddot{q})$

Then $\operatorname{IDM}\left(q_{d d m}\left(\hat{\chi}^{k}\right), \dot{q}_{d d m}\left(\hat{\chi}^{k}\right), \ddot{q}_{d d m}\left(\hat{\chi}^{k}\right)\right) \approx \operatorname{IDM}(q, \dot{q}, \ddot{q})$ for any $\hat{\chi}^{k}$, and the jacobian matrix (20) can be approximated by:

$\left(\frac{\partial\left(y_{S}\right)}{\partial \chi}\right)_{\hat{\chi}^{k}} \approx I D M\left(q_{d d m}\left(\hat{\chi}^{k}\right), \dot{q}_{d d m}\left(\hat{\chi}^{k}\right), \ddot{q}_{d d m}\left(\hat{\chi}^{k}\right)\right)$

Taking the approximation (22) of the jacobian matrix into the Taylor series expansion, it becomes:

$y=\tau=\operatorname{IDM}\left(q_{d d m}\left(\hat{\chi}^{k}\right), \dot{q}_{d d m}\left(\hat{\chi}^{k}\right), \ddot{q}_{d d m}\left(\hat{\chi}^{k}\right)\right) \chi^{k+1}+(o+e)$

This is the Inverse Dynamic Identification Model, IDIM, (6), where $(q, \dot{q}, \ddot{q})$ are estimated with $\left(q_{d d m}, \dot{q}_{d d m}, \ddot{q}_{d d m}\right)$, simulated from (17). At each iteration $k$, the IDIM method is applied as described in section 2. The sampling of (23) at a sampling rate $f_{m}$, gives the over-determined linear system:

$Y_{f m}(\tau)=W_{\delta f m}\left(q_{d d m}, \dot{q}_{d d m}, \ddot{q}_{d d m}, \hat{\chi}^{k}\right) \chi+\rho_{f m}$

The parallel decimation of (24) gives:

$Y(\tau)=W_{\delta}\left(q_{d d m}, \dot{q}_{d d m}, \ddot{q}_{d d m}, \hat{\chi}^{k}\right) \chi+\rho$

The LS solution of (25) calculates $\hat{\chi}_{k+1}$, at iteration $k+1$. This process converges in $n c$ steps such that:

$\left(\left\|\rho_{n c+1}\right\|-\left\|\rho_{n c}\right\|\right) /\left\|\rho_{n c}\right\| \leq$ tol $_{l}$ and $\left(\left\|\rho_{n c+1}\right\| /\|Y\|\right) \leq$ tol $_{2}$

$\mathrm{tol}_{1}$ and $\mathrm{tol}_{2}$ are values ideally chosen to be small numbers to get fast convergence with good accuracy.

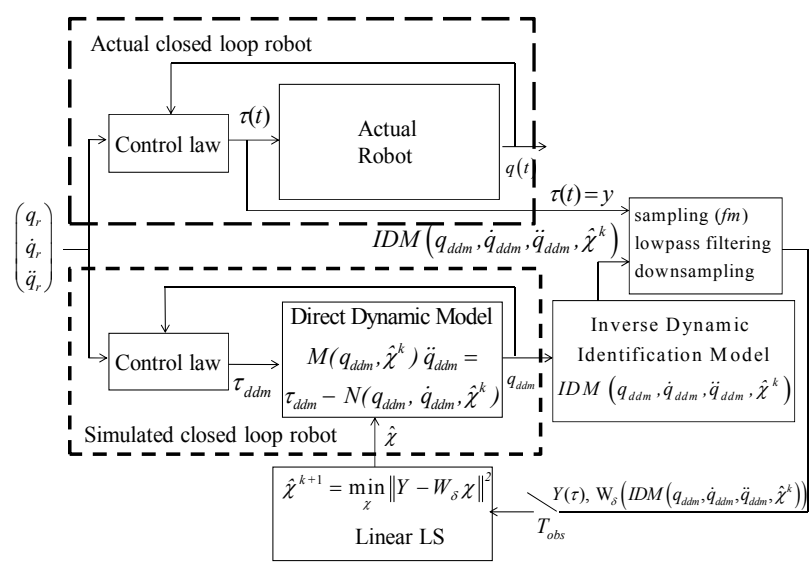

Fig. 2. DIDIM, with the Gauss-Newton regression, identification scheme.

Because this method uses both models DDM and IDIM, it is named the DIDIM method: Direct and Inverse Dynamic 
Identification Models technique. The DIDIM method with the Gauss-Newton regression is illustrated Fig. 2.

\section{B. Initialization of the algorithm}

A problem is how to choose the initial values $\hat{\chi}^{0}$. We can use CAD values, or identified values with the IDIM method, but we show that there is no need at all of a priori values. We propose an algorithm not sensitive to the initial conditions, which assumes that the condition $\left(q_{d d m}\left(\hat{\chi}_{k}\right), \dot{q}_{d d m}\left(\hat{\chi}_{k}\right), \ddot{q}_{d d m}\left(\hat{\chi}_{k}\right)\right) \simeq(q, \dot{q}, \ddot{q})$, is satisfied at any iteration $k$, and especially for $k=0$. This is a key point to assume the convergence of the Gauss-Newton regression.

This is possible by taking the same control law structure for the actual robot and for the simulated one with the same performances given by the bandwidth, the stability margin or the closed-loop poles. Because the simulated robot parameters $\hat{\chi}^{k}$, change at each iteration $k$, the gains of the simulated control law must be updated according to $\hat{\chi}^{k}$. We have shown that the actual control signal on joint $\mathrm{j}$ must be multiplied by $\frac{\hat{J}_{j}^{k}}{{ }^{a p} J_{j}}$ in order to obtain the same normalized second order $\frac{1}{s^{2}}$ open-loop system and the same closed-loop transfer function at each iteration $k$ [1]. This allows to keep $\left(q_{d d m}\left(\hat{\chi}_{k}\right), \dot{q}_{d d m}\left(\hat{\chi}_{k}\right), \ddot{q}_{d d m}\left(\hat{\chi}_{k}\right)\right) \simeq(q, \dot{q}, \ddot{q})$, at each iteration $k$.

$J_{j}$, is the maximum value, with respect to $q$, of the inertia moment around joint $z_{j}$ axis. This gives the smallest damping value and the smallest stability margin of the closed-loop second order transfer function, while $q$ varies.

${ }^{a p} J_{j}$ is an a priori value of the unknown actual values ${ }^{a} J_{j}$. It can be calculated from a priori CAD values of inertial parameters and must be taken at least as $Z Z_{j}+I_{a_{j}}$.

$\hat{J}_{j}^{k}$ is the estimated value of $J_{j}$ at iteration $k$.

We propose to take a regular inertia matrix $M\left(q_{d d m}, \hat{\chi}^{0}\right)$, in order to have a good initialization for the numerical integration of the DDM. This is named the "regular initialization". It can be obtained with:

$\hat{\chi}^{0}=0$, except for, $I a_{j}^{0}=1, j=1, n$

The inertia of the rotor and gear of actuator $j$ is generally taken into account in the IDM model (1) as: $\tau_{r_{j}}=I a_{j} \ddot{q}_{j}$

Then, the initial inertia matrix becomes the identity matrix, which is the best regular matrix:

$M\left(q_{d d m}, \hat{\chi}^{0}\right)=I_{n}$
Another point is to choose the state initial condition of the state vector, $\left(q_{d d m}(0), \dot{q}_{d d m}(0)\right)$, in order to integrate the DDM. Because DIDIM doesn't need the joint position measurement, the actual values $(q(0), \dot{q}(0))$, are supposed to be unknown and we choose, $\left(q_{d d m}(0), \dot{q}_{d d m}(0)\right)=\left(q_{r}(0), \dot{q}_{r}(0)\right), \quad$ which $\quad$ is close to $(q(0), \dot{q}(0))$. Because the closed-loop transient response due to different initial conditions differs between the actual and the simulated signals during a transient period of approximately, $5 /{ }^{d} \omega_{n}$, the corresponding joint force/torque samples are eliminated from the identification data in (24).

\section{CASE STUDY: MODELING OF THE TX40 ROBOT}

The Stäubli TX-40 robot has a serial structure with six rotational joints. The robot kinematics is defined using the modified Denavit and Hartenberg notation (Fig. 3).

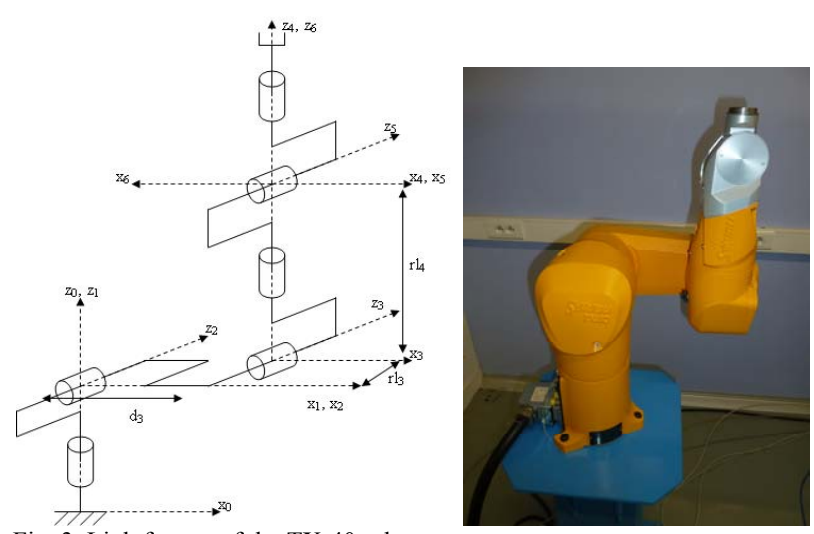

Fig. 3. Link frames of the TX-40 robot

TABLE 1 GEOMETRIC PARAMETERS OF THE TX-40 ROBOT
\begin{tabular}{|c|c|c|c|r|c|}
\hline$j$ & $\sigma_{j}$ & $\alpha_{j}$ & $d_{j}$ & $\theta_{j}$ & $r_{j}$ \\
\hline 1 & 0 & 0 & 0 & $\theta_{l}$ & 0 \\
\hline 2 & 0 & $-\pi / 2$ & 0 & $\theta_{2}$ & 0 \\
\hline 3 & 0 & 0 & $d 3=0.225 \mathrm{~m}$ & $\theta_{3}$ & $r l 3=0.035 \mathrm{~m}$ \\
\hline 4 & 0 & $\pi / 2$ & 0 & $\theta_{4}$ & $r l 4=0.225 \mathrm{~m}$ \\
\hline 5 & 0 & $-\pi / 2$ & 0 & $\theta_{5}$ & 0 \\
\hline 6 & 0 & $\pi / 2$ & 0 & $\theta_{6}$ & 0 \\
\hline
\end{tabular}

The geometric parameters defining the robot frames are given in Table 1 . The parameter $\sigma_{j}=0$, means that joint $\mathrm{j}$ is rotational, $\alpha_{j}$ and $d_{j}$ give respectively the angle and distance between $z_{j-1}$ and $z_{j}$ along $x_{j-1}$, whereas $\theta_{j}$ and $r_{j}$ give respectively the angle and distance between $x_{j-1}$ and $x_{j}$ along $z_{j}$. Since all the joints are rotational then $\theta_{j}$ is the position variable of joint $j$.

The TX40 robot is characterized by a coupling between the joints 5 and 6 such that $\left[\begin{array}{c}\dot{q} r_{5} \\ \dot{q} r_{6}\end{array}\right]=\left[\begin{array}{cc}K 5 & 0 \\ K 6 & K 6\end{array}\right]\left[\begin{array}{c}\dot{q}_{5} \\ \dot{q}_{6}\end{array}\right]$. Where $\dot{q} r_{j}$ is the velocity of the rotor of motor $j, \dot{q}_{j}$ is the velocity of joint $j, K 5$ is the transmission gain ratio of axis 5 and $K 6$ is the transmission gain ratio of axis 6 . Thus, the duality relation 
of force/torque gives $\left[\begin{array}{c}\tau_{c_{s}} \\ \tau_{c_{6}}\end{array}\right]=\left[\begin{array}{cc}K 5 & K 6 \\ 0 & K 6\end{array}\right]\left[\begin{array}{l}\tau_{r_{s}} \\ \tau_{r_{b}}\end{array}\right]$. Where, $\tau_{c j}$ is the motor's torque of joint $j$, taking into account the coupling effect, $\tau_{r j}$ is the electro-magnetic torque of the rotor of motor $j$. The coupling between joints 5 and 6 , also adds to the effect of the inertia of rotor 6 and new viscous and Coulomb friction parameters $f v_{m 6}$ and $f_{c_{m}}$ to both $\tau_{c 5}$ and $\tau_{c \sigma}$.

We can write:

$\tau_{c_{5}}=\tau_{5}+I a_{6} \ddot{q}_{6}+f v m_{6} \dot{q}_{6}+f c m_{6} \operatorname{sign}\left(\dot{q}_{6}\right)$

$\tau_{c_{6}}=\tau_{6}+I a_{6} \ddot{q}_{5}+f v m_{6} \dot{q}_{5}+f c m_{6} \operatorname{sign}\left(\dot{q}_{5}\right)$

Where $\tau_{5}, \tau_{6}$ already contain the terms $\left(\operatorname{Ia} \ddot{q}_{j}+f v_{j} \dot{q}_{j}+f c_{j} \operatorname{sign}\left(\dot{q}_{j}\right)\right)$, for $j=5$ and 6 respectively,

$I a_{5}=K_{5}^{2} J a_{5}+K_{6}^{2} J a_{6}$ and $I a_{6}=K_{6}^{2} J a_{6}$

$J a_{j}$ is the moment of inertia of rotor $j, f v_{m 6}, f c_{m 6}$ are the friction parameters due to the coupling between joints 5 and 6 . The TX40 has $N_{s}=86$, standard dynamic parameters given by the $14 * 6$ usual standard parameters (3), plus $f v_{m} 6$ and $f c_{m 6}$. For IDIM-LS method, we use the standard inverse dynamic model (2). The columns of the matrix $I D M_{s t}(q, \dot{q}, \ddot{q})$ in (2) can be obtained using the recursive algorithm of Newton-Euler. We use the software SYMORO+ to automatically calculate the customized symbolic expressions of the models [13]. The base parameters $\chi$ and the minimal model (4) are automatically calculated using a $Q R$ numerical method [11]. The matrices $M\left(q_{d d m}, \chi\right)$ and $N\left(q_{d d m}, \dot{q}_{d d m}, \chi\right)$ are numerically calculated using the IDM model $\tau_{i d m}(q, \dot{q}, \ddot{q})$ (1), for special values of $q, \dot{q}, \ddot{q}$.

\section{V.EXPERIMENTAL IDENTIFICATION RESULTS}

The identification of the dynamic parameters has been carried out using one trajectory using the controller CS8C of the Stäubli robots.
The joint positions and torques are stored with a sampling frequency measurement $f_{m}=5 \mathrm{KHz}$. The IDIM-LS off-line estimation is carried out with a filtered position $\hat{q}$, calculated with a $50 \mathrm{~Hz}$ cut-off frequency forward and reverse Butterworth filter, and with the velocities $\hat{\dot{q}}$, and the accelerations, $\hat{\ddot{q}}$, calculated with a central difference algorithm of $\hat{q}$. The parallel decimation of $Y_{f m}$ and $W_{f m}$, in (8), is carried out with a sample rate divided by a factor, $n_{d}=100$, and a lowpass filter cut-off frequency equal to, $0.8^{*} f_{m} /\left(2 * n_{d}\right)=20 \mathrm{~Hz}$. There are 60 base parameters which can be simplified to 23 well identified essential parameters with good relative standard deviation.

The DIDIM method is initialized with all the standard parameters equal 0 except $I_{a j}=1, j \neq 5$ and $\mathrm{I}_{\mathrm{a} 5}=2$, due to the coupling effect (29). The simulation Fig.4, is carried out with the actual stored reference trajectory and the CS8C controller of the TX40, with updating the gains with $\hat{J}_{j}^{k} /{ }^{a p} J_{j}$, and using the simulink software. A step of DIDIM takes $7^{\prime}$ on a 2008 PC working station.

The results are given in Table 2 .

\begin{tabular}{|c|l|l|l|l|l|}
\hline $\begin{array}{c}\text { Paramete } \\
\mathrm{r}\end{array}$ & $\hat{\chi}^{l}$ & $\% \sigma_{\hat{\chi}_{\mathrm{r}}}$ & $\begin{array}{c}\text { Paramete } \\
\mathrm{r}\end{array}$ & $\hat{\chi}^{l}$ & $\% \sigma_{\hat{\chi}_{\mathrm{r}}}$ \\
\hline$Z Z_{1 R}$ & 1.30 & 0.65 & $F v_{3}$ & 2.37 & 1.2 \\
\hline$F v_{1}$ & 8.71 & 0.8 & $F c_{3}$ & 6.6 & 2.0 \\
\hline$F c_{1}$ & 7.69 & 2.5 & $I a_{4}$ & 0.029 & 5.0 \\
\hline$X X_{2 R}$ & -0.53 & 3.6 & $F v_{4}$ & 1.0 & 1.2 \\
\hline$X Z_{2 R}$ & -0.16 & 7.2 & $F c_{4}$ & 2.47 & 2.2 \\
\hline$Z Z_{2 R}$ & 1.09 & 0.8 & $I a_{5}$ & 0.053 & 12.0 \\
\hline$M X_{2 R}$ & 2.11 & 0.4 & $F v_{5}$ & 2.52 & 2.3 \\
\hline$F v_{2}$ & 7 & 1.1 & $F c_{5}$ & 2.77 & 5.0 \\
\hline$F c_{2}$ & 7.74 & 1.7 & $F v_{6}$ & 0.72 & 2.7 \\
\hline$Z Z_{3 R}$ & 0.14 & 3.7 & $F c_{6}$ & 0.9 & 8.0 \\
\hline$M Y_{3 R}$ & -0.64 & 1.8 & $f v_{m 6}$ & 0.8 & 2.4 \\
\hline$I a_{3}$ & 0.083 & 7.1 & $f c_{m 6}$ & 1.6 & 4.4 \\
\hline
\end{tabular}

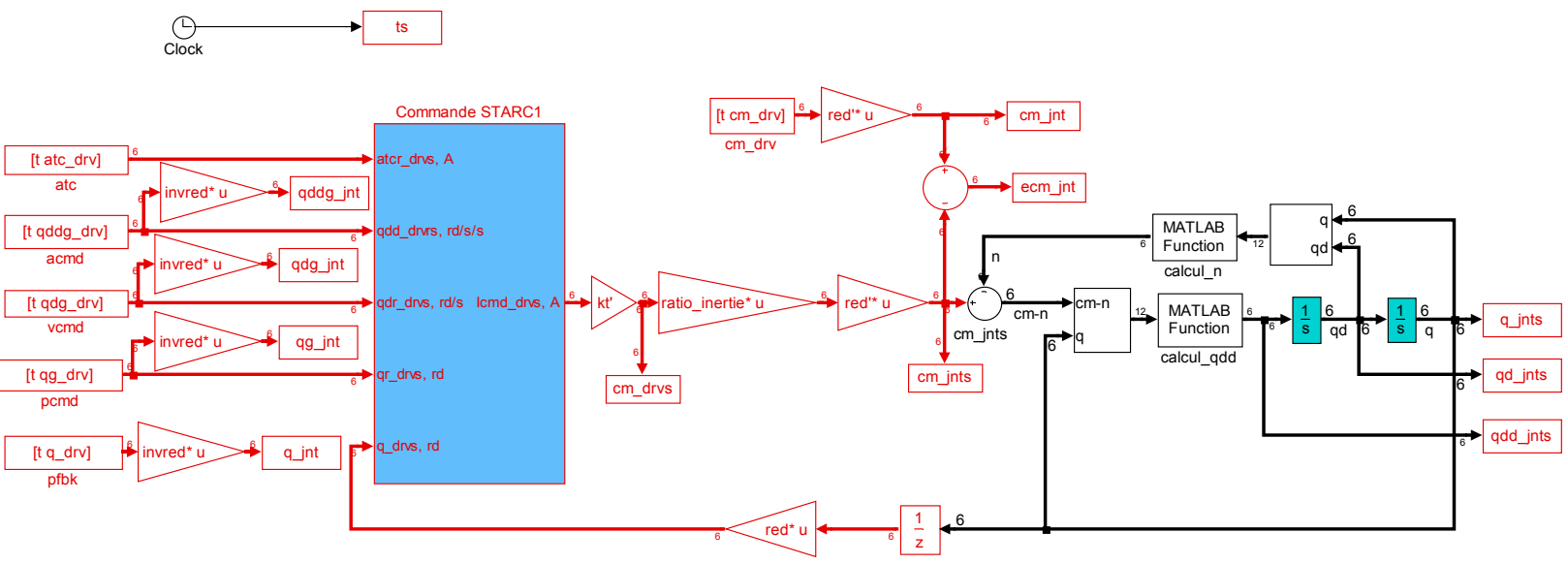

Fig. 4. Simulation of the TX_40 with simulink, red line: CS8C controller, black line: the direct dynamic model. 

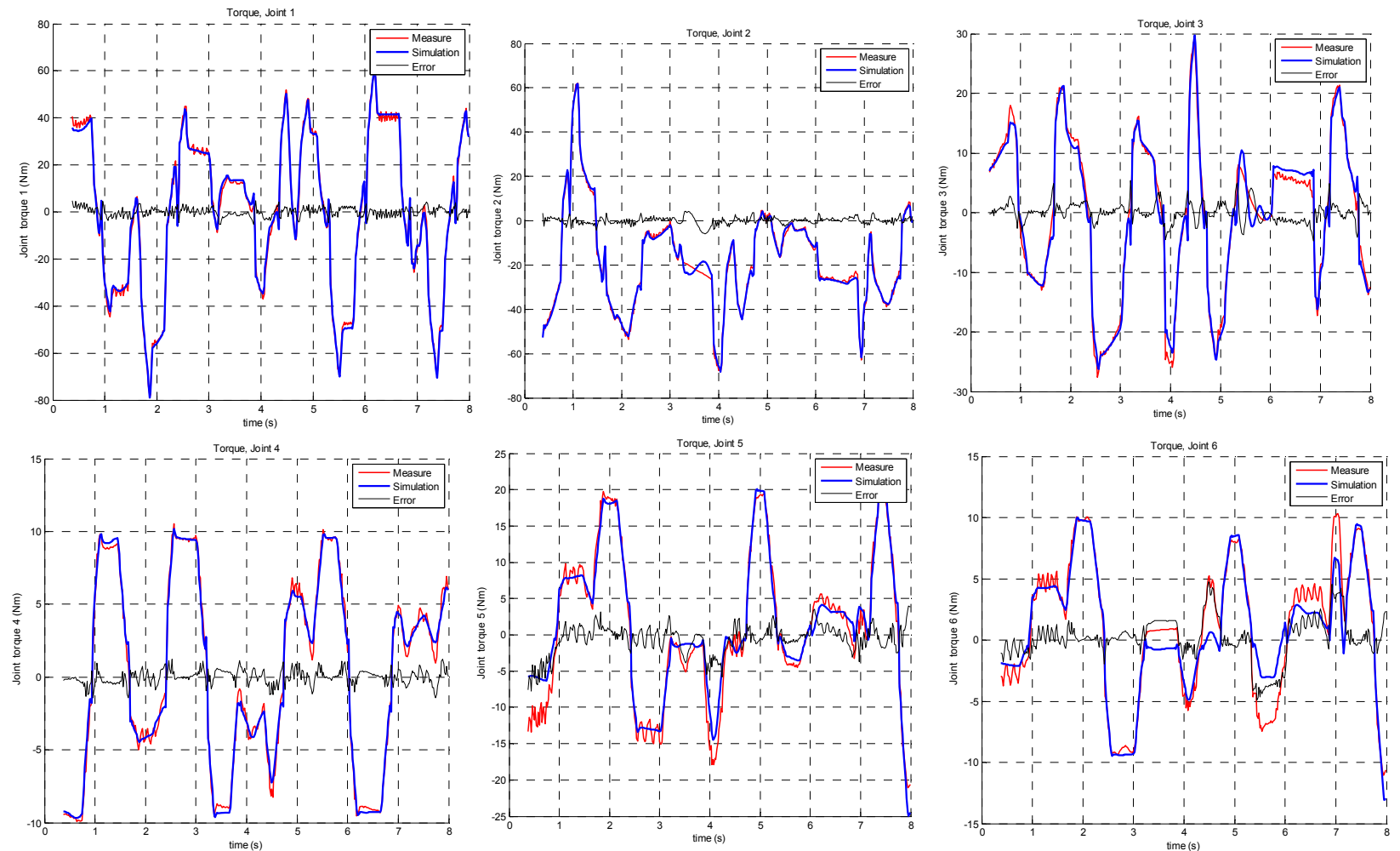

Fig. 5. DIDIM, validation, red line: actual torque, blue line: estimated torque, $Y_{e}=W_{\delta}\left(q_{d d m}, \dot{q}_{d d m}, \ddot{q}_{d d m}, \hat{\chi}^{I}\right) \hat{\chi}^{I}$, black line: error

Considering the test stop (26), it needs only 1 step to obtain the optimal solution which is very close to the IDIM solution. Hence, the DIDIM method has a very fast convergence. A validation is plotted on Fig.5 at the decimated frequency $50 \mathrm{~Hz}$. It shows that the actual joint torques, $Y(\tau)$, and the torques estimated with the identified model, $Y_{e}=W_{\delta}\left(q_{d d m}, \dot{q}_{d d m}, \ddot{q}_{d d m}, \hat{\chi}^{I}\right) \hat{\chi}^{l}$, as defined in (25) are very close. Both methods, IDIM-LS and DIDIM, give a small relative norm error, $\|Y-W \hat{\chi}\| /\|Y\|<3 \%$, which shows a good accuracy for the model and for the identified value.

\section{CONCLUSION}

This paper deals with a new off-line identification technique of robot dynamic parameters, called DIDIM for Direct and Inverse Dynamic Identification Models technique. This method is a closed-loop Output Error approach, considering the output is the joint force/torque. The optimal parameters are the solution of a non-linear least-squares problem which is solved with a Gauss-Newton method. Each step of the iterative procedure of the Gauss-Newton regression is dramatically simplified to a linear regression which is solved with the Inverse Dynamic Identification Model technique (IDIM). In this paper we prove that DIDIM is very efficient on a 6 dof industrial robot, with a 1 step convergence starting with a regular initialization of the parameters.

\section{REFERENCES}

[1] M. Gautier, A. Janot, and P. Vandanjon, "DIDIM: A new method for the dynamic identification of robots from only torque data," in Proc. of IEEE International Conference on Robotics and Automation, pp. 2122-2127, 2008.

[2] P. Khosla and T. Kanade, "Parameter Identification of Robot Dynamics," in Proc. of 24th IEEE Conference on Decision and Control, pp. 1754-1760, 1985.

[3] C. Atkeson, C. An, and J. Hollerbach, "Estimation of Inertial Parameters of Manipulator Loads and Links," Int. J. of Robotics Research, vol. 5, no. 3, pp. 101-119, 1986.

[4] M. Gautier, "Identification of Robot Dynamics," in Proc. of IFAC Symposium on Theory of Robots, pp. 351-356, 1986.

[5] H. Kawasaki and K. Nishimura, "Terminal-Link Parameter Estimation and Trajectory Control," IEEE Transactions on Robotics and Automation, vol. 4, no. 5, pp. 485-490, Oct. 1988.

[6] I. Ha, Ko, and Kwon, "An efficient estimation algorithm for the model parameters of robotic manipulators," IEEE Trans. on Robotics and Automation, vol. 5, no. 6, pp. 386-394, 1989.

[7] M. Gautier, "Dynamic Identification of Robots with Power Model," in Proc. of IEEE International Conference on Robotics and Automation, pp. 1922-1927, 1997.

[8] J. Swevers, W. Verdonck, and J. De Schutter, "Dynamic model identification for industrial robots - Integrated experiment design and parameter estimation," IEEE control systems magazine, vol. 27, no. 5 , pp. $58-71,2007$.

[9] J. Hollerbach, W. Khalil, and M. Gautier, "Model Identification," in Springer Handbook of Robotics, vol. 14, Springer, 2008.

[10] W. Khalil and E. Dombre, Modeling identification and control of robots. Taylor and Francis, 2002.

[11] M. Gautier, "Numerical calculation of the base inertial parameters," Journal of Robotics Systems, vol. 8, no. 4, pp. 485-506, 1991.

[12] M. Gautier and W. Khalil, "Exciting trajectories for the identification of the inertial parameters of robots," International Journal of Robotics Research, vol. 11, no. 4, pp. 362-375, Aug. 1992.

[13] W. Khalil and D. Creusot, "SYMORO+: A system for the symbolic modelling of robots," Robotica, vol. 15, pp. 153-161, 1997. 